Europhys. Lett., 55 (3), pp. 425-431 (2001)

\title{
Modeling the electrophoretic deposition of colloidal particles
}

\author{
A. T. PÉREZ ${ }^{1}$, D. SAVIlle ${ }^{2}$ and C. Soria ${ }^{1}$ \\ 1 Departamento de Electrónica y Electromagnetismo, Facultad de Física \\ Avda. Reina Mercedes s/n 41012 Sevilla, Spain \\ 2 Department of Chemical Engineering, Princeton University - Princeton, NJ 08544, USA
}

(received 18 December 2000; accepted in final form 25 May 2001)

PACS. 82.45.-h - Electrochemistry and electrophoresis.

PACS. 82.70.Dd - Colloids.

PACS. 47.65.+a - Electrohydrodynamics and magnetohydrodynamics.

\begin{abstract}
This letter presents the results of numerical simulations of the buildup of a layer of colloidal particles on an electrode. In a low-frequency electric field, particles suspended in a lowconductivity liquid migrate to one electrode and then to the other. During each cycle, deposits are formed and dissipated. The current-voltage characteristics of the process reflect properties of the suspension and the deposited layer. Using a flux corrected transport (FCT) algorithm, the transport equation for the particle phase is solved simultaneously with equations describing the electric field. The model reproduces the main features of the current-voltage relation.
\end{abstract}

Introduction. - Particles of various sorts often acquire charge when immersed in lowconducting liquids. Although the zeta potentials of such particles are usually smaller than those encountered in aqueous systems, the use of judiciously chosen additives contributes to the charge on the particles and helps stabilize the suspension. Moreover, some surfactant molecules form inverse micelles that stabilize ions and this, in turn, increases the conductivity of the liquid. This leads to charged particles with moderately thick double layers.

In this work we study the electrophoretic deposition of colloidal particles suspended in low-conducting liquids. The problem is relevant for fundamental as well as applied reasons. From the fundamental point of view, the mechanics of particle aggregation is related to the interparticle forces [1]. From the applied point of view, electrophoretic deposition is often used in liquid xerography to increase image quality [2].

In [1] a technique for measuring the cohesion of a layer of colloidal particles is described. According to the procedure, application of a certain voltage causes particle buildup on one electrode. When the polarity is reversed and voltage increased, the layer breaks. The voltages at which the layer is formed and broken are related to the consolidation and tensile strengths of the layer. Submitting the suspension to a very low-frequency alternating electric field causes a layer of aggregated particles to form, alternatively, on each electrode. A hysteresis loop is obtained when the electric current is plotted as a function of voltage. This loop is associated with the migration of particles. In this letter we present a simple model that reproduces the main features of the mechanisms responsible for the loop.

Model. - Consider a suspension of particles between two parallel electrodes a distance $L$ apart. The volume fraction of particles is denoted by $\phi$. The electrophoretic mobility of the particles is $K(\phi)$, so that the relative velocity of the particles to the liquid is $\boldsymbol{v}=K(\phi) \boldsymbol{E}, \boldsymbol{E}$ the electric field. The conductivity $\sigma(\phi)$ is a function of the volume fraction. The electrodes are infinity large and we place the $z$-axis perpendicular to them in such a way that $z=0$ and $z=L$ 
define the electrode planes. A time-dependent voltage, $V(t)$, is applied across the electrodes. We use the following scales: $L$ for length, $K_{0} V_{0} / L$ for velocity, $V_{0}$, the maximum applied voltage, for electric potential, $\sigma_{0} V_{0} / L$ for current density and $\epsilon V_{0} / L^{2}$ for charge density. Here $\epsilon$ is the dielectric constant and the reference values for mobility and conductivity correspond to the limits at infinite dilution: $K_{0}=K(0)$ and $\sigma_{0}=\sigma(0)$. The equation that determines the volume fraction $\phi(z, t)$ of particles is, in non-dimensional form [3]:

$$
\frac{\partial \phi}{\partial t}+\frac{\partial}{\partial z}(\phi(1-\phi) K(\phi) E)=\frac{1}{P e} \frac{\partial}{\partial z}\left(K_{\mathrm{h}}(\phi) \frac{\partial(\phi Z(\phi))}{\partial z}\right) ;
$$

the factor $(1-\phi)$ takes into account the back flow of the fluid necessary to compensate for the advance of particles towards the electrode. The Peclet number $P e$ is $K_{0} V_{0} / D_{0}$. The particle diffusion coefficient depends on the volume fraction, and can be related to the osmotic pressure as $D(\phi)=D_{0} K_{\mathrm{h}}(\phi) \frac{\mathrm{d}(\phi Z(\phi))}{\mathrm{d} \phi}$ with $Z(\phi)$ the non-dimensional osmotic pressure $Z(\phi)=$ $\Pi /\left(n k_{\mathrm{B}} T\right)$ ( $n$ is the number density of particles, $k_{\mathrm{B}}$ Boltzmann's constant and $T$ the absolute temperature). For very dilute systems $Z \rightarrow 1$ and the diffusion coefficient is constant, $D=D_{0}$. But as the volume fraction tends to a certain maximum packing fraction, the osmotic pressure diverges, reflecting the fact that the particles cannot be further compressed.

The coefficient $K_{\mathrm{h}}(\phi)$ is the hydrodynamic mobility and, although its relation to the electric mobility is straightforward in the case of ions, this is not the case for colloidal particles $\left({ }^{1}\right)$.

The electric field equations are

$$
\frac{\mathrm{d} E}{\mathrm{~d} z}=\rho, \quad \frac{\tau_{\mathrm{r}}}{\tau_{\mathrm{t}}} \frac{\partial \rho}{\partial t}+\frac{\mathrm{d}(\sigma E)}{\mathrm{d} z}=0, \quad V(t)=\int_{0}^{1} E \mathrm{~d} z,
$$

where we assume that quantities vary only in $z$ and $E(z)$ stands for the $z$ component of the electric field. All the quantities appearing in these equations are dimensionless. $\tau_{\mathrm{r}}$ and $\tau_{\mathrm{t}}$ denote, respectively, the electric relaxation time of the liquid $\left(\tau_{\mathrm{r}}=\epsilon / \sigma_{0}\right)$ and the time of flight of particles between the electrodes in a very dilute suspension $\left(\tau_{\mathrm{t}}=L^{2} / K_{0} V_{0}\right)$.

We focus on the process of layer formation, so the problem is one-dimensional and the fields depend only on $z$. Accordingly, the liquid motion is reduced to the back flow $-\phi \boldsymbol{v}$ and the equation of motion for the liquid need not be solved.

For a model suspension of $\mathrm{TiO}_{2}$ particles in mineral oil, typical values for the quantities involved are [1]: $\epsilon=1.6 \times 10^{-11} \mathrm{~F} / \mathrm{m}, \sigma_{0}=10^{-8} \mathrm{~S} / \mathrm{m}, K_{0}=3 \times 10^{-10} \mathrm{~m}^{2} / \mathrm{Vs}$ and $D_{0}=8 \times 10^{-14} \mathrm{~m}^{2} / \mathrm{s}$ (note that $K_{0} / D_{0} \gg e / k_{\mathrm{B}} T$ ). In the experiments typical values for $V_{0}$ and $L$ are $100 \mathrm{~V}$ and $1 \mathrm{~mm}$, respectively. These give $P e=400,000$ and $\tau_{\mathrm{r}} / \tau_{\mathrm{t}}=5 \times 10^{-4}$. Therefore, the diffusion term in eq. (1) and the time-depending term in the charge conservation eq. (2) are negligible.

With these approximations the equations become

$$
\frac{\partial \phi}{\partial t}+\frac{\partial}{\partial z}(\phi(1-\phi) K(\phi) E)=0
$$

and

$$
j(t)=\sigma(z) E(z), \quad V(t)=\int_{0}^{1} E \mathrm{~d} z,
$$

where $j(t)$ defines the electric current density.

Before going further, two important points deserve attention. The first one concerns the diffusion term in eq. (1). For large Peclet numbers this term is negligible, except when the osmotic pressure gradient becomes of order $P e$. This should be the case in the boundary

$\left({ }^{1}\right)$ If we were to study the sedimentation problem under gravity force, we would have $v=K_{\mathrm{h}}(\phi) U_{0}$ with $U_{0}$ the Stokes velocity. 
between the suspension and the aggregated layer. There, the osmotic pressure increases to prevent concentrations beyond the maximum packing. However, the extent of the region where diffusion must be taken into account is of order $1 / P e$ and we can safely divide the domain into two regions: the concentrated layer with $\phi=\phi_{\mathrm{m}}$, where $\phi_{\mathrm{m}}$ is the maximum volume fraction, and the suspension with $\phi<\phi_{\mathrm{m}}$. This can be easily accommodated by making $K(\phi) \rightarrow 0$ for $\phi \rightarrow \phi_{\mathrm{m}}$. Although there is no physical reason for imposing this condition on $K$, it assures that the net flux of particles towards the concentrate is zero and it produces the correct velocity for the discontinuity. Also the details of the layer formation are insensitive to the particular form of $K(\phi)$.

The second remark concerns the time-dependent electrical phenomena. Neglecting the first term in the charge conservation equation is accurate only if the temporal variation of the applied field is of order of, or slower than, the drift of particles between both electrodes. For example, if the voltage is $V(t)=\sin (2 \pi t / T)$ the approximation is valid only for $T \geq 1$. If we were interested in faster transients, the temporal derivative must be taken into account.

Qualitative behavior. - Consider eq. (3) with $E=1$ and the following initial and boundary conditions: $\phi(z=1, t)=\phi_{\mathrm{m}}, \quad \phi(z, t=0)=\phi_{0} \quad$ if $\quad 0<z<1$. The initial condition represents a homogeneous distribution of particles through the whole gap.

The solution of eq. (3) is obtained by the method of characteristics. Hence, $\phi$ is constant along the lines

$$
\frac{\mathrm{d} z}{\mathrm{~d} t}=\frac{\mathrm{d} f(\phi)}{\mathrm{d} \phi},
$$

where the flux function is $f(\phi)=\phi(1-\phi) K(\phi)$. It is more convenient to reformulate the boundary and initial conditions problem as an initial value problem [4]: $\phi(z, t=0)=\phi_{0}$ if $0<z<1, \phi(z, t=0)=\phi_{\mathrm{m}}$ if $1<z, \quad \phi(z, t=0)=0$ if $z<0$.

Figure 1 shows the characteristic lines. Since the volume fraction becomes multi-valuated at regions where the characteristics intersect, shock-like discontinuities develop. These discontinuities move with velocity given by $U_{\mathrm{s}}=\left(f\left(\phi_{+}\right)-f\left(\phi_{-}\right)\right) /\left(\phi_{+}-\phi_{-}\right)$, where the signs \pm denote the sides of the discontinuity. One discontinuity develops at the boundary between the clear liquid below, and the suspension above. It moves with velocity $U_{1}=f\left(\phi_{0}\right) / \phi_{0}=$ $\left(1-\phi_{0}\right) K\left(\phi_{0}\right)$. The other one is the boundary between the layer of aggregated particles and the suspension. This velocity is negative, and its absolute value is $U_{2}=f\left(\phi_{0}\right) /\left(\phi_{\mathrm{m}}-\phi_{0}\right)=$ $\left(1-\phi_{0}\right) \phi_{0} K\left(\phi_{0}\right) /\left(\phi_{\mathrm{m}}-\phi_{0}\right)$, where it has been used that $f\left(\phi_{\mathrm{m}}\right)=0$.

When both discontinuities meet, a new (stationary) discontinuity forms between the aggregate and the clear liquid. The final thickness of the aggregate, $\delta_{\mathrm{f}}=\phi_{0} / \phi_{\mathrm{m}}$, is reached at $t=t_{\mathrm{c}}=1 /\left(U_{1}+U_{2}\right)$. In the case $K(\phi)=1-\phi / \phi_{\mathrm{m}}$ it is $t_{\mathrm{c}}=1 /\left(1-\phi_{0}\right)$. Note that for $t<t_{\mathrm{c}}$ there are three regions of constant volume fraction: the layer with $\phi=\phi_{\mathrm{m}}$, the suspension with $\phi=\phi_{0}$ and the liquid with $\phi=0$. Figure 2 is a schematic view of these regions.

Clearly the time of formation of the layer is related to the particle mobility. Measuring the electric current during the transient, it is possible, experimentally, to determine this time and the electrophoretic mobility. Varying the initial volume fraction reveals its dependence on $\phi$. We recall that the standard techniques for measuring the electrophoretic mobility are usually valid for dilute suspensions. The measurement of the electric current during the layer formation for different volume fractions provides an alternative technique.

As a second problem consider the case when a layer has already been formed between $z=0$ and $z=\delta_{0}$ and a constant voltage is applied. In this case the initial conditions are: $\phi(z, t=$ $0)=\phi_{\mathrm{m}}$ if $0<z<\delta_{0}, \phi(z, t=0)=0$ if $\delta_{0}<z<1, \phi\left(z, t>t_{1}\right)=\phi_{\mathrm{m}}$ if $1<z, \phi(z, t=0)=0$ if $z<0$, where $t_{1}=\left(1-\delta_{0}\right) / f^{\prime}(0)$ is the time at which the first characteristic emanating from $z=\delta_{0}$ reaches the upper electrode. At this moment the layer starts to build up at this electrode. Figure 3 shows a schematic view of the characteristics. From the point $z=\delta_{0}$, 


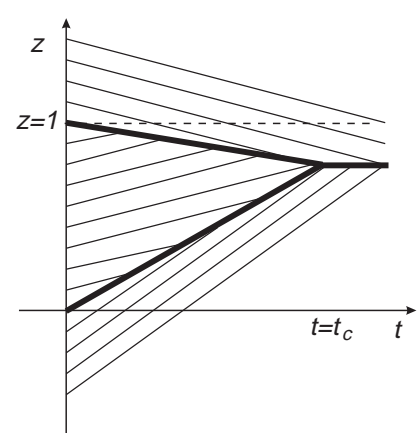

Fig. 1

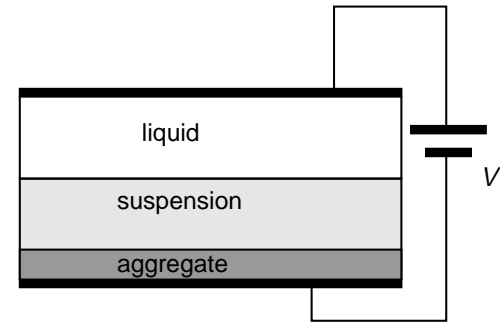

Fig. 2

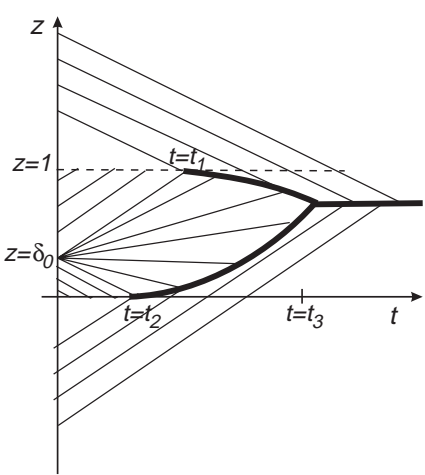

Fig. 3

Fig. 1 - Characteristic curves and associated discontinuities for the formation of a layer from a homogeneous suspension.

Fig. 2 - Schematic representation of the formation of a layer under an applied voltage.

Fig. 3 - Characteristic curves and associated discontinuities for the transfer of a layer from one electrode to the other.

$t=0$ emanates a fan of lines that fill the gap between the characteristics, with slopes $f^{\prime}(0)$ and $f^{\prime}\left(\phi_{\mathrm{m}}\right)$. This structure is analogous to a rarefaction wave in gases. Time $t_{2}=\delta_{0} / f^{\prime}\left(\phi_{\mathrm{m}}\right)$ corresponds to the arrival of the characteristic emanating from $z=\delta_{0}$ at the lower electrode. From here a shock separating clear fluid from suspension develops. On the other hand, a shock separating the layer of aggregate from the suspension develops from $z=1$ at $t=t_{1}$. Now, both discontinuities are curved, as a consequence of the continuous variation of the volume fraction. At a certain time $t_{3}$, for which the analytical expression is rather cumbersome, both discontinuities merge in the final one that separates the clear liquid from the aggregate.

Mobility and conductivity of concentrated suspensions. - The electrophoretic mobility of particles has been a subject of research for many years and expressions for the mobility of spherical particles with double layers of arbitrary thickness in very dilute suspensions are available. However, there are no simple closed form expressions for concentrated suspensions. Although many papers deal with the electrophoretic mobility for concentrated suspensions, there is no general agreement on the relation between mobility and volume fraction. It appears that hydrodynamic and electrical interactions balance each other for the case of very thin double layers $[5,6]$. In that case the electrophoretic mobility will be insensitive to particle concentration up to moderate values of the volume fraction. For human erythrocytes, Zukoski and Saville [7] found that this is the case up to volume fractions close to 1 for deformable particles.

The situation is similar for the conductivity where, strictly speaking, the Maxwell expression applies only to dilute suspensions of uncharged particles. The effective conductivity of dilute suspensions of charged particles has been addressed by Saville [8]. In this work the effect of the counterions and non-specific adsorption is taken into account. The expression for the conductivity is $\sigma=\sigma_{0}(1+\alpha \phi)$, where the coefficient $\alpha$ ranges between 1 and 60 depending on the zeta potential and the double-layer thickness. A rough estimate of the conductivity of the suspension is

$$
\sigma=\sigma_{0}+n_{\mathrm{c}} z_{\mathrm{c}} e K_{\mathrm{c}}+n_{\mathrm{p}} z_{\mathrm{p}} e K_{0}
$$

where $n_{\mathrm{c}}$ is the average number density of counterions, $z_{\mathrm{c}}$ the valence of the counterions, $K_{\mathrm{c}}$ their mobility, $n_{\mathrm{p}}$ the density of particles, $z_{\mathrm{p}}$ the number of elementary charges per particle and $K_{0}$, as above, the electrophoretic mobility of the particles at infinite dilution. For a suspension of $\mathrm{TiO}_{2}$ particles in mineral oil [1] $\sigma_{0}=10^{-8} \mathrm{~S} / \mathrm{m}, K_{\mathrm{c}}=5 \times 10^{-10} \mathrm{~m}^{2} / \mathrm{Vs}$, $K_{0}=3 \times 10^{-10} \mathrm{~m}^{2} / \mathrm{Vs}, n_{\mathrm{p}}=3 \times 10^{18} \phi \mathrm{m}^{-3}, z_{\mathrm{c}}=1$ and $z_{\mathrm{p}}=30$. Introducing $n_{\mathrm{c}}=\left(z_{\mathrm{p}} / z_{\mathrm{c}}\right) n_{\mathrm{p}}$ 


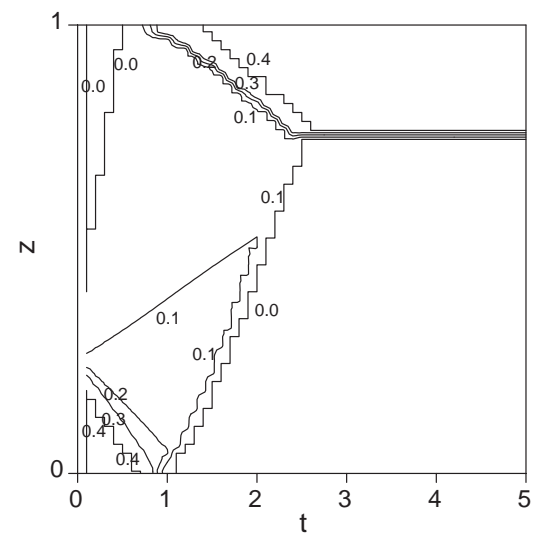

Fig. 4 - Curves of constant volume fraction for the transfer of a layer from one electrode to the other. For $1<t<2$ a region with a continuous variation of $\phi$ is observable.

we obtain the estimate $\sigma=\sigma_{0}(1+1.2 \phi)$. We have to note that in this case we are dealing with a non-aqueous liquid and the values of zeta potential obtained in such systems are smaller than in aqueous systems.

In what follows we suppose that both $K(\phi)$ and $\sigma(\phi)$ are known. Lacking well-established expressions, we have chosen simple, reasonable forms for these functions. Since the conductivity has more influence on the current than the mobility during particle sedimentation, we paid more attention to the role of the conductivity.

Numerical solution. - We solved eq. (3) for the volume fraction using a Flux Corrected Transport (FCT) algorithm, which is widely used to solve problems in fluid dynamics that involve shocks and discontinuities. There is a variety of FCT algorithms and since our problem is a transient, one-dimensional problem, we have used the one adapted by Morrow and Cram [9]. To simulate a condition of zero flux at the boundaries we have introduced two false nodes at each boundary and imposed symmetrical volume fractions there with respect to $z=0$ and $z=1$.

To compute the electric field it is necessary to evaluate the integral in eq. (4), taking into account the spatial dependence of the conductivity induced by the variation of the volume fraction. The integral is computed using a cubic spline interpolation for the values of $\sigma(\phi(z))$. In all the computations shown below, the distance between nodes is $\Delta z=0.01$ and the time step used $\Delta t=0.001$. The functions $K(\phi)$ and $\sigma(\phi)$ can be varied as desired.

Deposition from homogeneous suspension. - To test to the overall algorithm, we solved the problem of a homogeneous suspension subject to a constant voltage with $K(\phi)=1-\phi / \phi_{\mathrm{m}}$ and $\sigma(\phi)=1$. The maximum and initial values of the volume fraction are $\phi_{\mathrm{m}}=0.4$ and $\phi_{0}=0.1$. The contour plot of the volume fraction as a function of $z$ and $t$ corresponds exactly to that expected from fig. 1. The two expected discontinuities are clear, one separating the layer of the aggregate from the uniform suspension, the other separating the suspension from the clear liquid. Since $t_{\mathrm{c}}=1 /\left(1-\phi_{0}\right)$, we predict a time of 1.1 for the merging of the two initial discontinuities, in total agreement with the numerical solution. The final sediment has a thickness of 0.25 , corresponding to $\phi_{0} / \phi_{\mathrm{m}}$.

Transit of a layer between two electrodes. - A second calculation simulates the transfer of a layer of particles from one electrode to the other when a constant voltage is applied. Keeping the same mobility dependence, we used $\sigma=1+\alpha \phi_{0}$, with $\alpha=5$. The initial layer is between $z=0$ and $z=0.25$, with the maximum concentration $\phi=0.4$. Figure 4 is a contour plot of the volume fraction in this case. As expected, two curved discontinuities merge into 


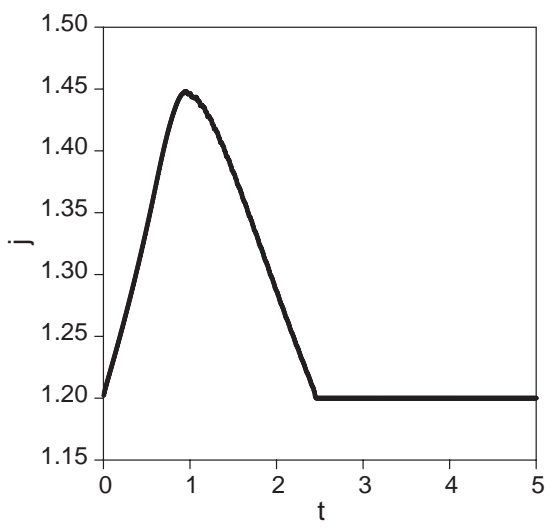

Fig. 5

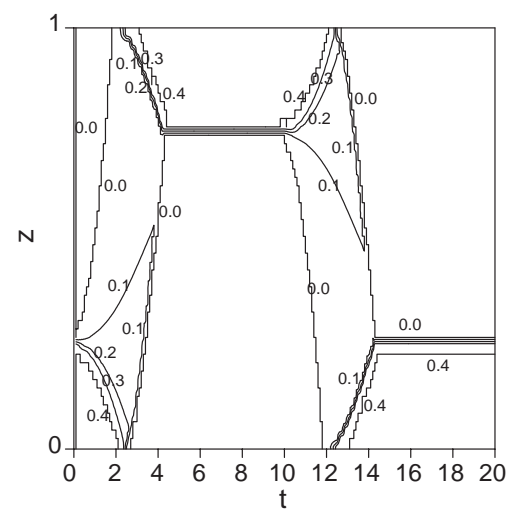

Fig. 6

Fig. 5 - Electric current as a function of time for the transfer of the layer from one electrode to the other.

Fig. 6 - Curves of constant volume fraction during a cycle for a time-dependent voltage $V(t)=$ $\sin (2 \pi t / T)$.

the final steady-state discontinuity, that represents the layer of particles formed on the other electrode. A fan of constant volume fraction curves emanates from $z=0.25$ at $t=0$ according to the picture described above. This fan of curves results, for $t \sim 1$, in a continuous variation of the volume fraction between 0 and 0.4 through the gap. Later the layer begins to built onto the upper electrode and for $t=2.5$ the final steady-state is reached. Figure 5 shows the current density as a function of time. The current when the layer is formed is 1.2 , according to eqs. (4). The maximum current recorded is close to the value 1.5 , corresponding to a homogeneous suspension. The coefficient $\alpha$ determines the contribution of particles to the current. The greater $\alpha$, the higher the current peak. The electric field is higher where the conductivity is lower. When the layer is formed onto the upper electrode, the electric field is constant in the two regions, the clear liquid region and the layer, but has different values on each zone. The discontinuity of the electric field gives place to a surface charge density at the interface. This surface charge density will play a major role in the electrohydrodynamic stability of the advancing particle front.

Motion under a low-frequency alternating voltage. - Finally, we consider the simulation of a complete cycle of sinusoidal voltage. This case is of interest because this variation of the

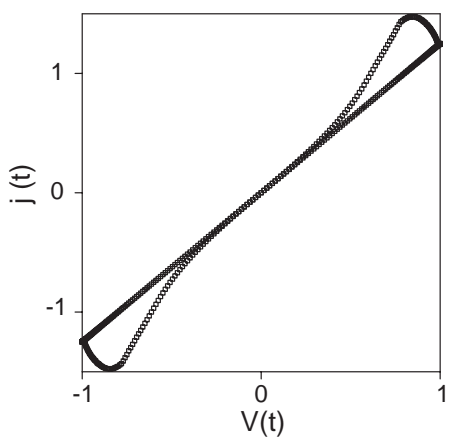

Fig. 7

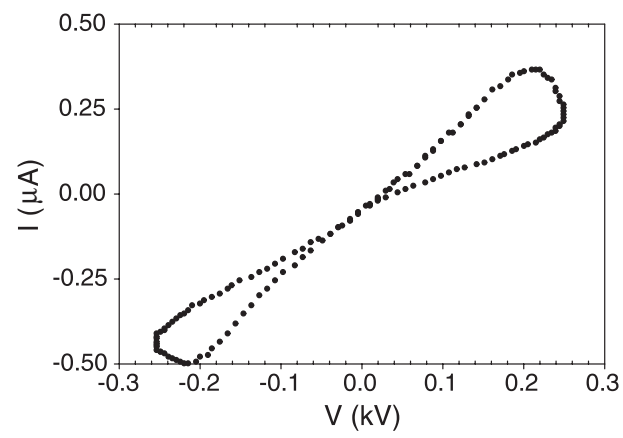

Fig. 8

Fig. 7 - Electric current as a function of voltage during a period for a low-frequency electric field.

Fig. 8 - Measured current vs. applied voltage for a xerographic ink. The period of the signal is $100 \mathrm{~s}$, its amplitude $250 \mathrm{~V}$. (Adapted from [1].) 
electric current was used experimentally to determine the mechanical stresses of the aggregates $[1,2]$. Starting with a layer of maximum volume fraction on one electrode, we apply a voltage $V(t)=\sin (2 \pi t / T)$. After the initial transient, the layer will be transferred back and forth from one electrode to the other. Figure 6 shows constant volume fraction lines for a complete cycle. In this plot we used $K(\phi)=\left(1-\phi / \phi_{\mathrm{m}}\right), \sigma=1+10 \phi, \phi_{\mathrm{m}}=0.4, \delta_{0}=0.25$ and $T=20$. The successive formation of the layer at both electrodes is clear in the figure. The distinct feature from these simulations is that the characteristics start at $t=0$ with a zero slope, corresponding to a zero electric field. Figure 7 is a plot of the current as a function of the applied voltage. The loop observed in this curve has the same qualitative behavior as those observed experimentally (see fig. 8). When the voltage is increasing, the layer is being built and an additional contribution to the current is noticeable. Once the layer is formed, the current is linearly dependent on the voltage. When the voltage becomes negative, the particles begin to migrate towards the other electrode, making an additional contribution to the negative current. This contribution disappears once the layer is assembled.

The amplitude of the loops is related to the coefficient $\alpha$ in the conductivity function. For $\alpha=1$ the loop is scarcely noted, whereas for $\alpha=10$ it is clearly visible. Taking into account that the correction to the liquid conductivity is $\alpha \phi$ and that $\phi$ is of order 0.2 , this is equivalent to saying that, to have a noticeable effect on the current, the contribution of the particles to the effective conductivity must be greater than the liquid conductivity itself. For $\mathrm{TiO}_{2}$ particles in mineral oil used in [1] the loop is small, in agreement with our estimate of $\alpha \sim 1$ for this suspension. On the other hand, the xerographic ink used in the same work presents the loop plotted in fig. 8, indicating a greater value of $\alpha$ for this suspension.

Conclusion. - We presented a simple model for the electrophoretic deposition of colloidal particles. The model relies on the knowledge of the mobility $K(\phi)$, and conductivity $\sigma(\phi)$. The model reproduces the loops in the current-voltage characteristics that are obtained experimentally when a suspension of colloidal particles is subjected to a low-frequency AC voltage. To obtain the wide loops observed experimentally, we showed that the contribution of the particles to the conductivity of the suspension has to be greater than the liquid conductivity itself. It is also shown that $K(\phi)$ is related to the time of formation of a layer of particles from a homogeneous suspension; we suggest how simple deposition experiments may be used to determine this relationship. To bring theory and experiment into closer agreement will require better knowledge of how mobility and conductivity depend on volume fraction.

$$
* * *
$$

This work was carried out with financial support from Ministerio de Ciencia y Tecnología, BFM2000-1056.

\section{REFERENCES}

[1] Lemaire E., Merhi D., Pérez A. T. and Valverde J. M., to be published in J. Electrostatics.

[2] Chang S., Till H., Viturro E., Watson K., Pérez A. T., González A. and Castellanos A., NIP14 International Conference on Digital Printing Technologies, Toronto, 1998.

[3] Russel W. B., Saville D. A. and Schowalter W. R., Colloidal Dispersions (Cambridge University Press) 1989.

[4] Bustos M. C. and Concha F., Sedimentation of Small Particles in a Viscous Fluid, edited by Tory E. M. (Computational Mechanics Publications) 1996.

[5] Acrivos A., Jeffrey D. J. and Saville D. A., J. Fluid Mech., 212 (1990) 95.

[6] Reed L. D. and Morrison Jr. F. A., J. Colloid Interface Sci., 54 (1976) 117.

[7] Zukoski IV C. F. and Saville D. A., J. Colloid Interface Sci., 115 (1987) 422.

[8] Saville D. A., J. Colloid Interface Sci., 91 (1983) 34.

[9] Morrow R. and Cram L. E., J. Comput. Phys., 57 (1985) 129. 